\title{
A Virtual Reality Safety and Hazard Analysis Simulation
}

\author{
John T. Bell and H. Scott Fogler \\ University of Illinois, Chicago / University of Michigan, Ann Arbor
}

\section{Abstract}

A virtual reality based chemical plant simulation has been developed for the study of safety and hazard analysis in undergraduate chemical engineering. The simulation focuses on a pilot plant scale polyether polyol production facility, and also includes relevant features of the nearby environment. The virtual plant is based upon information and photographs collected from a real nearby facility, but is not intended to accurately represent any real facility.

In the original implementation, students were given a written description of the facility ( including process chemistry and MSDS sheets ), and asked to explore the virtual plant to identify both safety concerns and examples of safety systems in use. They were then asked to write an analysis of the overall safety of the plant, with recommendations for improvement. A comparative study of students using virtual reality versus students who based their analysis solely on the written process description did not show the desired benefits, and therefore the simulation has recently been revised.

The new version provides more directed student activity, through the use of specific questions to be addressed during the simulation, as well as a scoring system to provide additional incentive. Students must find and activate question-mark icons, which trigger full-screen photographic images and auditory explanations of the situations at hand. Students must then select a red, yellow, or green light, to indicate whether the situation is dangerous, cautionary, or safe, respectively.

\section{Introduction and Background}

Virtual reality, VR, is an emerging computer interface that employs three-dimensional interactive immersive computer graphics, along with psychological and cognitive methodologies and special interface devices, to invoke a strong sense of presence or 'being there' to users. Although the ideal goal of a simulation indistinguishable from reality[1] has not yet been achieved, many simulations have been developed that deliver very strong positive impact on users[2-7].

The authors of this paper have been working for several years to develop applications of VR to undergraduate chemical engineering education[8-12], with goals that include the determination of the optimal applicability of VR to undergraduate engineering education. Among the findings is the conclusions that VR is best used in situations where the real thing is unavailable (due to physical, economic, logistical, or other constraints ), and in which 3-D viewing and/or a sense of presence provide significant benefits. One area that fits these criteria extremely well is the area of safety, wherein hazardous environments can be safely "experienced" using VR. This paper describes a simulation that applies VR to safety education - the hazard analysis of a chemical production facility - and how it has been recently improved. 


\section{Process \& Plant Description}

The process featured in this simulation is a pilot-plant scale polyether-polyol production facility, located on a river near a major metropolitan city in the upper Midwest United States. Because a proper hazard evaluation should also consider the nearby environment, the simulation includes nearby homes and businesses as well as the production facility and support buildings. The most highly detailed area is inside the pilot plant, where reactors, scrubber systems, fire suppression systems and other equipment are all modeled with as much detail as is practically possible for educational VR. ( Increased detail decreases execution speed. There is a fine balance to be achieved for simulations that must run on student-affordable personal computers. )

( It should be noted that although the simulation uses photographs and other information taken from real chemical plants, the resulting simulation is not intended to be an accurate representation of any real facility. In particular, all proprietary information has been either omitted or altered, and additional hazards have been introduced into the simulation that are not present in the actual plants. )

The chemistry of polyether polyol production generally involves a polymerization reaction of ethylene oxide or propylene oxide with multifunctional glycols, such as shown in Figure 1:

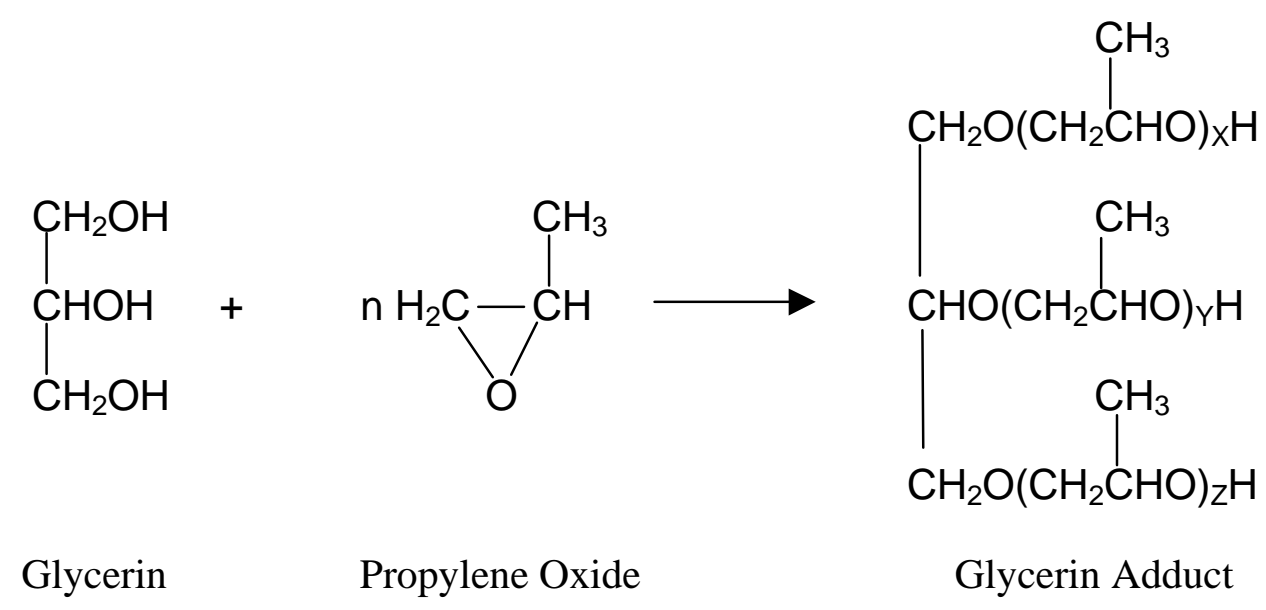

Figure 1: Typical Polyether-Polyol Chemistry

The oxides are especially hazardous materials, because in addition to being both toxic and flammable, they have an upper flammability limit of $100 \%$, which means that they contain enough oxygen in their own structures to support combustion, and will burn in the absence of any external oxidant. (Inerting is insufficient to prevent combustion of these materials.) Polymerization reactions are also well known as hazardous situations, and special care must be taken to prevent and control runaway reactions, and to make sure that venting and drain systems are capable of handling viscous polymeric materials. 


\section{Initial Results}

The initial implementation of this simulation asked students to explore the pilot plant and its environs, and to prepare an evaluation of the hazards and safety systems present, along with their recommendations for improvements. In addition to VR, students were provided with a written description of the facility and reaction chemistry, including material safety data sheet (MSDS ) information for all chemicals present. (The VR simulation also includes an interactive help feature, which provides additional information and equipment photographs on request. )

A two-year study was performed involving 300 total students, split into two groups each year. Half of the students performed their evaluations using both the VR simulation and the written description, while the other half used only the written description. The student hazard evaluations were then compared to determine the effect of the VR simulation.

The results included some notable papers in which the VR students made astute observations that would not have been possible based solely on the written description, and in which a clear understanding of the three-dimensional layout of the plant played an important role. However, the overall data did not show a statistically significant difference between the two groups. By contrast, a small number of students were taken to the real chemical plant, and their evaluations were notably superior to the rest of the class. This last result only goes to show that real reality will always be better than virtual reality, when it is a viable option. On the other hand, it should also be noted that this opportunity could only be offered to fifteen students out of a class of 150 ( one year only ), and that only ten of these fifteen were able to fit the trip into their schedules, ( mostly by skipping other classes. )

\section{Interactive Refinements}

Our conclusion from the above results was that the original implementation did not provide students with sufficient directed activity. Students let loose in a real chemical plant without any guidance and merely told to "look around" probably would not learn much from that experience either. It was therefore decided to modify the simulation, to provide specific issues for the students to consider, and a scoring system to provide incentive for careful thought.

The simulation was augmented with a series of "question mark" icons distributed throughout the chemical plant and surrounding areas, with each icon corresponding to a different specific situation for the students to evaluate. When a student clicks on one of the question marks, a photographic image fills the screen, and an auditory narration describes the situation at hand. ( Some scenarios entail multiple images and sounds played in series; Some provision has been made for movies or other media as opposed to still images, but that feature has not been fully implemented. ) After the scenario has been presented, a series of three spheres appear next to the question mark - one each of red, yellow, and green in a standard traffic light configuration, as shown in Figure 2. ( To be readily recognizable by color-blind individuals. Note that the electronic version of this paper contains full-color images that may not reproduce well on blackand-white printers. ) 

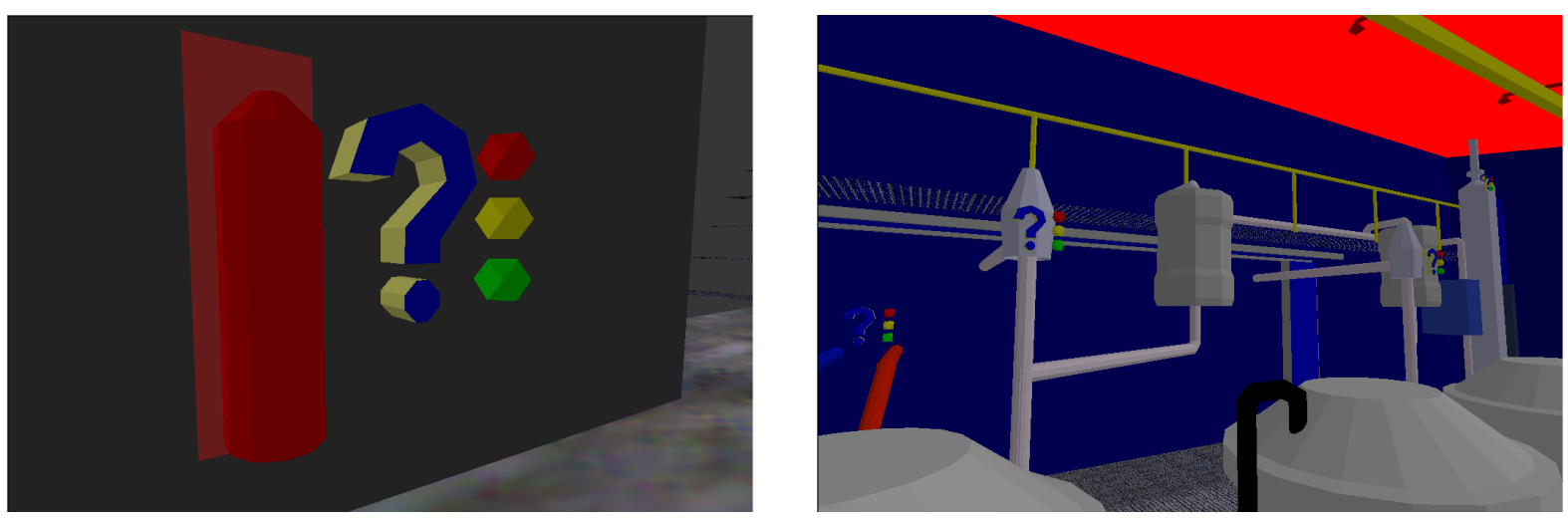

Figure 2: Sample Images Showing Question Mark Icons in the Chemical Plant

At this point the student must evaluate the scenario and select one of the three choices:

Red - Danger: The situation presented is hazardous, quite likely to cause or aggravate an accident if not corrected. (Examples: unvented storage tanks containing volatile components, undiked tanks adjacent to the river, and employees drinking water from a sink in the production area. )

Yellow - Caution: There are some dangers present, which must be carefully considered. However, the overall situation is neither especially dangerous nor exemplary. ( Examples: housing located adjacent to the chemical plant, and a grating floor for the upper levels of the pilot plant. )

Green - Exemplary: This is an example of an effective safety system in action. ( Examples: Double block and bleed feed control system, sprinkler systems, process pressure-relief vent systems. )

\section{Scoring System}

The computer program maintains a "score" that is continuously updated and displayed on the bottom edge of the screen, along with counters indicating how many questions have been found and/or answered and how many remain. Students receive one point for finding and activating each question mark, and four points if they select the correct response on their first try, making each question worth a maximum of five points. If the students' first choice is incorrect, then they can receive two points for selecting the correct response from the remaining two choices; No points are awarded for determining the answer by process of elimination. Students can repeat questions and answers as many times as they wish, however no points are awarded for repeat selections. The question data is all stored in a plain ASCII text file, such that the number and makeup of the questions in the simulation can be easily changed. For our purposes, there are twenty questions for a total value of 100 points. 


\section{Recommendations for Use}

The purpose of this simulation is not so much to teach new information or to test students' knowledge, but rather to stimulate reflective thoughts and discussion. It is therefore recommended that students go through the simulation in small groups of two or three, so that they can discuss and debate the scenarios before ( and after) selecting a response. Some of the scenarios lie in gray areas, and students (or instructors) may disagree with the answers provided. These questions can then provide the basis for a larger classroom discussion and debate. There is no reason to put heavy emphasis on the score, as students could easily repeat the simulation to earn a perfect score with no educational gain.

\section{Acknowledgements}

The authors wish to gratefully acknowledge the efforts of the undergraduate student programmers who have assisted in the development of the module described here, specifically Paul Sonda, Anita Sujarit, Scott Whitney, James Angelo, Jessica DesNoyer, Kim Snodgrass, Candace Corea, Christine Cha, and Matt Moersfelder ( in chronological order. ) Thanks are also due to Dr. Joseph Louvar and Lawrence James of BASF Chemical Corporation and to Tom Pakula of Marathon Oil Company for the valuable resources they have provided for the development of the virtual reality modules, and to the Department of Chemical Engineering of the University of Michigan for providing the initial funding of this project.

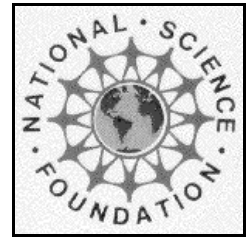

This project was supported, in part

by the

\section{National Science Foundation}

Opinions expressed are those of the authors and not necessarily those of the Foundation

\section{Bibliographical Information}

1. Sutherland, Ivan, " The Ultimate Display", Proceedings of the IFIP Congress, 1965.

2. Brooks, Frederick P., Jr., et al., "Project GROPE - Haptic Displays for Scientific Visualization", Computer Graphics: Proceedings of SIGGRAPH '90, 24(4), pp 177-185, August 1990.

3. Byron, S. and C. Levit. "The Virtual Windtunnel: An Environment for the Exploration of ThreeDimensional Unsteady Flows". Proceedings of IEEE Visualization '91, 1991.

4. Emerson, Toni and Debra Revere, "Virtual Reality in Training and Education: Resource Guide to Citations and Online Information", HITL Technical Publications, B-94-1 ( 1997 Revise ) 1994.

5. Pimental, Ken and Kevin Teixeira, Virtual Reality: Through the New Looking Glass. Second Edition, Windcrest Books, 1995.

6. Staff, "Immersive VR Tests Best", CyberEdge Journal, 4(6), November / December 1994.

7. Youngblut, Christine, "Educational Uses of Virtual Reality Technology", Technical Report IDA Document D-2128, 1998.

8. Bell, John T., "Virtual Reality in Chemical Engineering Laboratory Web Site", http://www.engin.umich.edu/labs/vrichel.

9. Bell, John T. and H. Scott Fogler. "Virtual Laboratory Accidents Designed to Increase Safety Awareness". Proceedings of American Society for Engineering Education Annual Conference, Charlotte, NC, American Society for Engineering Education, 1999, Best Paper Award. 
10. Bell, John T. and H. Scott Fogler. "Virtual Reality in Chemical Engineering Education". Proceedings of 1998 North Central Sectional ASEE Conference, University of Detroit Mercy, American Society for Engineering Education North Central Section, 1998.

11. Bell, John T. and H. Scott Fogler. "Recent Developments in Virtual Reality Based Education". Proceedings of American Society for Engineering Education Annual Conference, Washington, D.C., American Society for Engineering Education, 1996.

\section{Biographical Information}

\section{John T. Bell}

( Lecturer, Dept. of Electrical Engineering and Computer Science, University of Illinois, Chicago, 312 413-9054, jbell@eecs.uic.edu, http://www.eecs.uic.edu/ jbell, formerly at U of MI. ) Dr. Bell holds graduate degrees in Chemical Engineering and Computer Science, and combines both skills by developing computer simulations of chemical engineering related phenomenon. Recent work has focused on the application of interactive immersive three-dimensional display technology (a.k.a. VR) to the presentation of scientific, engineering, and educational information.

\section{H. Scott Fogler}

( Vennema Distinguished Professor of Chemical Engineering, University of Michigan, H.Scott.Fogler@umich.edu, http://www.engin.umich.edu/dept/che/people/fogler.html. ) Dr. Fogler is the author of four books and over 160 research publications. Awards include the ASEE Meriam-Wiley Distinguished Author Award (1996), the University of Michigan Distinguished Faculty Achievement Award (1996), the AIChE Warren K. Lewis Award (1995), and the Chemical Manufacturer's Association National Catalyst Award (1999). 